\title{
Pacific
}

Journal of

Mathematics

\section{A NOTE ON LAGRANGIAN COBORDISMS BETWEEN \\ LEGENDRIAN SUBMANIFOLDS OF $\mathbb{R}^{2 n+1}$}

\author{
ROMAN GOLOVKO
}




\title{
A NOTE ON LAGRANGIAN COBORDISMS BETWEEN LEGENDRIAN SUBMANIFOLDS OF $\mathbb{R}^{2 n+1}$
}

\author{
ROMAN GOLOVKO
}

\begin{abstract}
We study the relation of an embedded Lagrangian cobordism between two closed, orientable Legendrian submanifolds of $\mathbb{R}^{2 n+1}$. More precisely, we investigate the behavior of the Thurston-Bennequin number and (linearized) Legendrian contact homology under this relation. The result about the Thurston-Bennequin number can be considered as a generalization of the result of Chantraine which holds when $n=1$. In addition, we provide a few constructions of Lagrangian cobordisms and prove that there are infinitely many pairs of exact Lagrangian cobordant and not pairwise Legendrian isotopic Legendrian $n$-tori in $\mathbb{R}^{2 n+1}$.
\end{abstract}

\section{Introduction}

Basic definitions. A contact manifold $(M, \xi)$ is a $(2 n+1)$-dimensional manifold $M$ equipped with a smooth maximally nonintegrable hyperplane field $\xi \subset T M$, that is, locally $\xi=\operatorname{ker} \alpha$, where $\alpha$ is a 1-form which satisfies $\alpha \wedge(d \alpha)^{n} \neq 0$. $\xi$ is a contact structure and $\alpha$ is a contact 1-form which locally defines $\xi$. The Reeb vector field $R_{\alpha}$ of a contact form $\alpha$ is uniquely defined by the conditions $\alpha\left(R_{\alpha}\right)=1$ and $d \alpha\left(R_{\alpha}, \cdot\right)=0$. The most basic contact manifold is $\left(\mathbb{R}^{2 n+1}, \xi\right)$, where $\mathbb{R}^{2 n+1}$ has coordinates $\left(x_{1}, y_{1}, \ldots, x_{n}, y_{n}, z\right)$, and $\xi$ is given by $\alpha=d z-\sum_{i=1}^{n} y_{i} d x_{i}$. Note that $R_{\alpha}=\partial_{z}$. From now on, for ease of notation, we write $\mathbb{R}^{2 n+1}$ instead of $\left(\mathbb{R}^{2 n+1}, \xi\right)$.

A Legendrian submanifold of $\mathbb{R}^{2 n+1}$ is an $n$-dimensional submanifold $\Lambda$ which is everywhere tangent to $\xi$, that is, $T_{x} \Lambda \subset \xi_{x}$ for every $x \in \Lambda$. The Lagrangian projection is a map $\Pi: \mathbb{R}^{2 n+1} \rightarrow \mathbb{R}^{2 n}$ defined by

$$
\Pi\left(x_{1}, y_{1}, \ldots, x_{n}, y_{n}, z\right)=\left(x_{1}, y_{1}, \ldots, x_{n}, y_{n}\right) .
$$

Moreover, for $\Lambda$ in an open dense subset of all Legendrian submanifolds with $C^{\infty}$ topology, the self-intersection of $\Pi(\Lambda)$ consists of a finite number of transverse double points. Legendrian submanifolds which satisfy this property are called

MSC2010: primary 53D12; secondary 53D42.

Keywords: Legendrian submanifold, Lagrangian cobordism, Thurston-Bennequin number,

Legendrian contact homology. 
chord generic. A Reeb chord of $\Lambda$ is a path along the flow of the Reeb vector field which begins and ends on $\Lambda$. Since $R_{\alpha}=\partial_{z}$, there is a one-to-one correspondence between Reeb chords of $\Lambda$ and double points of $\Pi(\Lambda)$. From now on we assume that all Legendrian submanifolds of $\mathbb{R}^{2 n+1}$ are connected and chord-generic.

The symplectization of $\mathbb{R}^{2 n+1}$ is the symplectic manifold $\left(\mathbb{R} \times \mathbb{R}^{2 n+1}, d\left(e^{t} \alpha\right)\right)$, where $t$ is a coordinate on $\mathbb{R}$.

Definition 1.1. Let $\Lambda_{-}$and $\Lambda_{+}$be two Legendrian submanifolds of $\mathbb{R}^{2 n+1}$. We say that $\Lambda_{-}$is cobordant to $\Lambda_{+}$if there exists a smooth cobordism $\left(L ; \Lambda_{-}, \Lambda_{+}\right)$, and an embedding from $L$ to $\left(\mathbb{R} \times \mathbb{R}^{2 n+1}, d\left(e^{t} \alpha\right)\right)$ such that

$$
\begin{gathered}
\left.L\right|_{\left(-\infty,-T_{L}\right] \times \mathbb{R}^{2 n+1}}=\left(-\infty,-T_{L}\right] \times \Lambda_{-}, \\
\left.L\right|_{\left[T_{L}, \infty\right) \times \mathbb{R}^{2 n+1}}=\left[T_{L}, \infty\right) \times \Lambda_{+}
\end{gathered}
$$

for some $T_{L} \gg 0$ and $L^{c}:=\left.L\right|_{\left[-T_{L}-1, T_{L}+1\right] \times \mathbb{R}^{2 n+1}}$ is compact. In the case of a Lagrangian (exact Lagrangian) embedding, we say that $\Lambda_{-}$is Lagrangian (exact Lagrangian) cobordant to $\Lambda_{+}$. We will in general not distinguish between $L$ and $L^{c}$ and call both $L$.

From now on we assume that all embedded cobordisms in the symplectization of $\mathbb{R}^{2 n+1}$ are orientable.

We next define some notations. If $L$ is an embedded, embedded Lagrangian, or embedded exact Lagrangian cobordism from $\Lambda_{-}$to $\Lambda_{+}$, we write

$$
\Lambda_{-} \prec_{L} \Lambda_{+}, \quad \Lambda_{-} \prec_{L}^{\text {lag }} \Lambda_{+}, \quad \text { or } \Lambda_{-} \prec_{L}^{\mathrm{ex}} \Lambda_{+},
$$

respectively. If $L_{\Lambda}$ is a filling, Lagrangian filling, or exact Lagrangian filling of $\Lambda$ in the symplectization of $\mathbb{R}^{2 n+1}$, that is, $L_{\Lambda}$ is an embedded, embedded Lagrangian, or embedded exact Lagrangian cobordism with empty $-\infty$-boundary and $+\infty$ boundary $\Lambda$, then we write $\varnothing \prec_{L_{\Lambda}} \Lambda, \varnothing \prec_{L_{\Lambda}}^{\text {lag }} \Lambda$ or $\varnothing \prec_{L_{\Lambda}}^{\text {ex }} \Lambda$, respectively.

For the discussion about Lagrangian cobordisms between Legendrian knots, we refer to [Chantraine 2010; Ekholm et al. $\geq 2013$ ], and for the obstructions to the existence of Lagrangian cobordisms defined using the theory of generating families, we refer to [Sabloff and Traynor 2010; Sabloff and Traynor 2011].

Legendrian contact homology. Legendrian contact homology was independently introduced by Eliashberg, Givental, and Hofer [Eliashberg et al. 2000] and, for Legendrian knots in $\mathbb{R}^{3}$, by Chekanov [2002]. We now briefly remind the reader of the definition of the linearized Legendrian contact homology complex of a closed, orientable, chord-generic Legendrian submanifold $\Lambda \subset \mathbb{R}^{2 n+1}$; for more details see [Ekholm et al. 2005a].

Let $\mathscr{b}$ be the set of Reeb chords of $\Lambda$. Since $\Lambda$ is generic, $\mathscr{C}$ is a finite set. Let $A_{\Lambda}$ be the vector space over $\mathbb{Z}_{2}$ generated by the elements of $\mathscr{C}$ and $\mathscr{A}_{\Lambda}$ the unital 
tensor algebra over $A_{\Lambda}$, that is,

$$
A_{\Lambda}=\bigotimes_{k=0}^{\infty} A_{\Lambda}^{\otimes k}
$$

$\mathscr{A}_{\Lambda}$ is a differential graded algebra whose grading is denoted by $|\cdot|$ and whose differential is denoted by $\partial_{\Lambda}$. $\mathscr{A}_{\Lambda}$ is called a Legendrian contact homology differential graded algebra of $\Lambda$. For the definitions of $|\cdot|$ and $\partial_{\Lambda}$ we refer to Section 2 of [Ekholm et al. 2005b].

Note that it is difficult to use Legendrian contact homology in practical applications, as it is the homology of an infinite dimensional noncommutative algebra with a nonlinear differential. One of the ways to extract useful information from the Legendrian contact homology differential graded algebra is to follow Chekanov's [2002] linearization method, which uses an augmentation $\varepsilon: \mathscr{A}_{\Lambda} \rightarrow \mathbb{Z}_{2}$ to produce a finite-dimensional chain complex $\operatorname{LC}^{\varepsilon}(\Lambda)$ whose homology is denoted by $\operatorname{LCH}^{\varepsilon}(\Lambda)$. More precisely, $\varepsilon$ is a graded algebra map $\varepsilon: \mathscr{A}_{\Lambda} \rightarrow \mathbb{Z}_{2}$ that satisfy the following two conditions:

(1) $\varepsilon(1)=1$;

(2) $\varepsilon \circ \partial_{\Lambda}=0$.

Consider the graded isomorphism $\varphi^{\varepsilon}: \mathscr{A}_{\Lambda} \rightarrow \mathscr{A}_{\Lambda}$ defined by $\varphi^{\varepsilon}(c)=c+\varepsilon(c)$. This map defines a new differential $\partial^{\varepsilon}(c):=\varphi^{\varepsilon} \circ \partial_{\Lambda} \circ\left(\varphi^{\varepsilon}\right)^{-1}(c)$ and $\operatorname{LC}^{\varepsilon}(\Lambda):=\left(A_{\Lambda}, \partial_{1}^{\varepsilon}\right)$, where $\partial_{1}^{\varepsilon}: A_{\Lambda} \rightarrow A_{\Lambda}$ is a 1-component of $\partial^{\varepsilon}$. We let $\operatorname{LCH}_{\varepsilon}(\Lambda)$ be the homology of the dual complex $\operatorname{LC}_{\varepsilon}(\Lambda):=\operatorname{Hom}\left(\operatorname{LC}^{\varepsilon}(\Lambda), \mathbb{Z}_{2}\right)$.

Following Ekholm [2008], we observe that exact Lagrangian cobordism between two Legendrian submanifolds can be used to define a map between the Legendrian contact homology algebras.

In this paper, we establish the following two long exact sequences.

Theorem 1.2. Let $\Lambda_{-}$and $\Lambda_{+}$be two closed, orientable Legendrian submanifolds of $\mathbb{R}^{2 n+1}$ such that $\varnothing \prec_{L_{\Lambda_{-}}}^{\mathrm{ex}} \Lambda_{-}$. Then from the condition $\Lambda_{-} \prec_{L}^{\mathrm{ex}} \Lambda_{+}$it follows that there is an exact sequence

$$
\begin{aligned}
\rightarrow H_{i}\left(\Lambda_{-}\right) \rightarrow H_{i}(L) \oplus \mathrm{LCH}_{\varepsilon_{-}}^{n-i+2}\left(\Lambda_{-}\right) & \\
& \rightarrow \mathrm{LCH}_{\varepsilon_{+}}^{n-i+2}\left(\Lambda_{+}\right) \rightarrow H_{i-1}\left(\Lambda_{-}\right) \rightarrow .
\end{aligned}
$$

In addition, $\Lambda_{-} \prec_{L}^{\mathrm{ex}} \Lambda_{+}$implies that there is an exact sequence

$$
\begin{aligned}
\rightarrow \mathrm{LCH}_{\varepsilon_{-}}^{n-i+2}\left(\Lambda_{-}\right) \rightarrow \mathrm{LCH}_{\varepsilon_{+}}^{n-i+2}\left(\Lambda_{+}\right) & \\
& \rightarrow H_{i}\left(L, \Lambda_{-}\right) \rightarrow \mathrm{LCH}_{\varepsilon_{-}}^{n-i+3}\left(\Lambda_{-}\right) \rightarrow .
\end{aligned}
$$


Here $\mathrm{LCH}_{\varepsilon_{ \pm}}^{i}\left(\Lambda_{ \pm}\right)$is the linearized Legendrian contact cohomology of $\Lambda_{ \pm}$over $\mathbb{Z}_{2}$, linearized with respect to the augmentation $\varepsilon_{ \pm}$. $\varepsilon_{-}$is the augmentation induced by $L_{\Lambda_{-}}$, and $\varepsilon_{+}$is the augmentation induced by $L$ and $\varepsilon_{-}$.

We thank Joshua Sabloff and Lisa Traynor for pointing out how to get the second long exact sequence in Theorem 1.2.

The Thurston-Bennequin invariant. The Thurston-Bennequin invariant (number) of a closed, orientable, connected Legendrian submanifold $\Lambda$ of $\mathbb{R}^{2 n+1}$ was independently defined for $n=1$ by Bennequin [1983] and by Thurston, and was generalized to the case when $n \geq 1$ by Tabachnikov [1988].

Pick an orientation on $\Lambda \subset \mathbb{R}^{2 n+1}$. Push $\Lambda$ slightly off of itself along $R_{\alpha}=\partial_{z}$ to get another oriented submanifold $\Lambda^{\prime}$ disjoint from $\Lambda$. The Thurston-Bennequin invariant of $\Lambda$ is the linking number

$$
\operatorname{tb}(\Lambda)=\operatorname{lk}\left(\Lambda, \Lambda^{\prime}\right) .
$$

Note that $\operatorname{tb}(\Lambda)$ is independent of the choice of orientation on $\Lambda$, since changing it also changes the orientation of $\Lambda^{\prime}$.

Our goal is to prove the following theorem.

Theorem 1.3. Let $\Lambda_{-}$and $\Lambda_{+}$be two closed, orientable Legendrian submanifolds of $\mathbb{R}^{2 n+1}$.

(1) If $n$ is even and $\Lambda_{-} \prec_{L} \Lambda_{+}$,

$$
\operatorname{tb}\left(\Lambda_{+}\right)+\operatorname{tb}\left(\Lambda_{-}\right)=(-1)^{n / 2+1} \chi(L) .
$$

(2) If $n$ is odd, $\varnothing \prec_{L_{\Lambda_{-}}}^{\mathrm{ex}} \Lambda_{-}$, and $\Lambda_{-} \prec_{L}^{\mathrm{ex}} \Lambda_{+}$,

$$
\operatorname{tb}\left(\Lambda_{+}\right)-\operatorname{tb}\left(\Lambda_{-}\right)=(-1)^{((n-2)(n-1)) / 2+1} \chi(L) .
$$

Constructions and examples. Chantraine [2010] described the way to construct Lagrangian cobordisms from Legendrian isotopies of Legendrian knots. We show that the construction of Chantraine works in high dimensions. More precisely, we prove the following:

Proposition 1.4. Let $\Lambda_{-}, \Lambda_{+}$be two closed, orientable Legendrian submanifolds of $\mathbb{R}^{2 n+1}$ that are Legendrian isotopic. Then there exists an exact Lagrangian cobordism L such that

$$
\Lambda_{-} \prec_{L}^{\mathrm{ex}} \Lambda_{+}
$$

Front spinning is a procedure invented by Ekholm, Etnyre, and Sullivan [Ekholm et al. 2005b] to construct a closed, orientable Legendrian submanifold $\Sigma \Lambda \subset \mathbb{R}^{2 n+3}$ from a closed, orientable Legendrian submanifold $\Lambda \subset \mathbb{R}^{2 n+1}$. We will provide a detailed description of this procedure in Section 4 , and prove the following property of it. 
Proposition 1.5. Let $\Lambda_{-}, \Lambda_{+}$be two closed, orientable Legendrian submanifolds of $\mathbb{R}^{2 n+1}$. If $\Lambda_{-} \prec_{L}^{\operatorname{lag}} \Lambda_{+}$, there exists a Lagrangian cobordism $\Sigma L$ such that

$$
\Sigma \Lambda_{-} \prec_{\Sigma L}^{\operatorname{lag}} \Sigma \Lambda_{+}
$$

In addition, if $\Lambda_{-} \prec_{L}^{\mathrm{ex}} \Lambda_{+}$, there exists an exact Lagrangian cobordism $\Sigma L$ such that $\Sigma \Lambda_{-} \prec_{\Sigma L}^{\mathrm{ex}} \Sigma \Lambda_{+}$.

Finally, we apply Proposition 1.5 to the exact Lagrangian cobordisms from [Ekholm et al. $\geq 2013$ ] and construct exact Lagrangian cobordisms between the nonisotopic Legendrian tori described in [Ekholm et al. 2005b].

Proposition 1.6. There are infinitely many pairs of exact Lagrangian cobordant and not pairwise Legendrian isotopic Legendrian $n$-tori in $\mathbb{R}^{2 n+1}$.

\section{Proof of Theorem 1.2}

Proof. In this section, we prove the existence of the long exact sequences described in Theorem 1.2. We first construct an exact Lagrangian filling of $\Lambda_{+}$.

Since $\Lambda_{-}$is connected, and $L, L_{\Lambda_{-}}$are exact Lagrangian cobordisms in the symplectization of $\mathbb{R}^{2 n+1}$ such that the $(-\infty)$-boundary of $L$, which is $\Lambda_{-}$, agrees

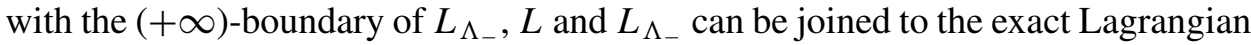
cobordism $L_{\Lambda_{+}}$in the symplectization of $\mathbb{R}^{2 n+1}$, where $L_{\Lambda_{+}}$is obtained by gluing the positive end of $L_{\Lambda_{-}}$to the negative end of $L$. Since the $-\infty$-boundary of $L_{\Lambda_{-}}$ is empty, the $-\infty$-boundary of $L_{\Lambda_{+}}$is also empty.

We now use the Mayer-Vietoris long exact sequence for $L_{\Lambda_{-}}, L \subset L_{\Lambda_{+}}$. We extend $L_{\Lambda_{-}}$and $L$ in such a way that $L_{\Lambda_{-}} \cap L$ is diffeomorphic to $\mathbb{R} \times \Lambda_{-}$. Hence the Mayer-Vietoris long exact sequence can be written as

$$
\rightarrow H_{i}\left(\mathbb{R} \times \Lambda_{-}\right) \rightarrow H_{i}(L) \oplus H_{i}\left(L_{\Lambda_{-}}\right) \rightarrow H_{i}\left(L_{\Lambda_{+}}\right) \rightarrow H_{i-1}\left(\mathbb{R} \times \Lambda_{-}\right) \rightarrow .
$$

Now we note that $H_{i}\left(\mathbb{R} \times \Lambda_{-}\right) \simeq H_{i}\left(\Lambda_{-}\right)$for all $i$. Hence we can rewrite the Mayer-Vietoris long exact sequence as

$$
\rightarrow H_{i}\left(\Lambda_{-}\right) \rightarrow H_{i}(L) \oplus H_{i}\left(L_{\Lambda_{-}}\right) \rightarrow H_{i}\left(L_{\Lambda_{+}}\right) \rightarrow H_{i-1}\left(\Lambda_{-}\right) \rightarrow .
$$

We now remind the reader of the following fact, which comes from certain observations of Seidel in wrapped Floer homology [Abouzaid and Seidel 2010; Fukaya et al. 2009].

Fact 2.1 [Ekholm 2012]. Let $\Lambda$ be a closed, orientable, connected, chord-generic Legendrian submanifold of $\mathbb{R}^{2 n+1}$ and $\varnothing \prec_{L_{\Lambda}}^{\mathrm{ex}} \Lambda$. Then

$$
H_{n-i+2}\left(L_{\Lambda}\right) \simeq \operatorname{LCH}_{\varepsilon}^{i}(\Lambda) .
$$

Here $\varepsilon$ is the augmentation induced by $L_{\Lambda}$. 
For the definition of the augmentation induced by a filling, we refer to Section 3 of [Ekholm 2008]. Also, [Ekholm 2012] provides a fairly complete sketch of a proof of Fact 2.1.

We change the indices in (2-2) and write it as

$$
H_{i}\left(L_{\Lambda_{ \pm}}\right) \simeq \operatorname{LCH}_{\varepsilon_{ \pm}}^{n-i+2}\left(\Lambda_{ \pm}\right) .
$$

Using (2-3), we rewrite the Mayer-Vietoris long exact sequence (2-1) as

$$
\begin{aligned}
\rightarrow H_{i}\left(\Lambda_{-}\right) \rightarrow H_{i}(L) \oplus \mathrm{LCH}_{\varepsilon_{-}}^{n-i+2}\left(\Lambda_{-}\right) & \\
& \rightarrow \mathrm{LCH}_{\varepsilon_{+}}^{n-i+2}\left(\Lambda_{+}\right) \rightarrow H_{i-1}\left(\Lambda_{-}\right) \rightarrow .
\end{aligned}
$$

We now write the long exact sequence for the pair $\left(L_{\Lambda_{-}}, L_{\Lambda_{+}}\right)$

$$
\rightarrow H_{i}\left(L_{\Lambda_{-}}\right) \rightarrow H_{i}\left(L_{\Lambda_{+}}\right) \rightarrow H_{i}\left(L_{\Lambda_{+}}, L_{\Lambda_{-}}\right) \rightarrow H_{i-1}\left(L_{\Lambda_{-}}\right) \rightarrow \text {. }
$$

Using (2-3) and the excision theorem for $L_{\Lambda_{+}}, L \subset L_{\Lambda_{+}}$, we write the long exact sequence (2-5) as

$$
\begin{aligned}
\rightarrow \mathrm{LCH}_{\varepsilon_{-}}^{n-i+2}\left(\Lambda_{-}\right) \rightarrow \mathrm{LCH}_{\varepsilon_{+}}^{n-i+2}\left(\Lambda_{+}\right) & \\
& \rightarrow H_{i}\left(L, \Lambda_{-}\right) \rightarrow \mathrm{LCH}_{\varepsilon_{-}}^{n-i+3}\left(\Lambda_{-}\right) \rightarrow .
\end{aligned}
$$

Remark 2.2. Under the conditions of Theorem 1.2, if $H_{i}\left(\Lambda_{-}\right)=H_{i-1}\left(\Lambda_{-}\right)=0$ for some $i$, say when $\Lambda_{-}=S^{n}$ and $i, i-1 \neq 0, n$, then long exact sequence (2-4) implies that

$$
\mathrm{LCH}_{\varepsilon_{+}}^{n-i+2}\left(\Lambda_{+}\right) \simeq H_{i}(L) \oplus \mathrm{LCH}_{\varepsilon_{-}}^{n-i+2}\left(\Lambda_{-}\right) .
$$

Hence, for such $i$, we get

$$
H_{i}(L) \simeq \mathrm{LCH}_{\varepsilon_{+}}^{n-i+2}\left(\Lambda_{+}\right) / \mathrm{LCH}_{\varepsilon_{-}}^{n-i+2}\left(\Lambda_{-}\right) .
$$

Remark 2.3. We can rewrite the long exact sequences (2-4) and (2-6) using the relative symplectic field theory of $\left(\left(\mathbb{R} \times \mathbb{R}^{2 n+1}, d\left(e^{t} \alpha\right)\right), L_{\Lambda_{ \pm}}\right)$, since

$$
E_{1}^{i}\left(\left(\mathbb{R} \times \mathbb{R}^{2 n+1}, d\left(e^{t} \alpha\right)\right), L_{\Lambda_{ \pm}}\right) \simeq \operatorname{LCH}_{\varepsilon_{ \pm}}^{i}\left(\Lambda_{ \pm}\right)
$$

over $\mathbb{Z}_{2}$. For the definition of the relative symplectic field theory, we refer to [Ekholm 2008], and for the details about the isomorphism described in (2-7), we refer to [Ekholm 2012]. (We observe that since $L_{\Lambda_{ \pm}}$are connected, the associated spectral sequences have only one level.)

\section{Proof of Theorem 1.3}

Let $n$ be even. We recall the following result: 
Proposition 3.1 [Eliashberg 1990]. Let $\Lambda$ be a closed, orientable, connected, chord-generic Legendrian submanifold of $\mathbb{R}^{2 n+1}$, where $n$ is even. Then

$$
\operatorname{tb}(\Lambda)=(-1)^{n / 2+1} \frac{1}{2} \chi(\Lambda) .
$$

We now note that

$$
\chi(\partial L)=2 \chi(L),
$$

since the Euler characteristic of an even-dimensional boundary is twice the Euler characteristic of its bounded manifold; see Chapter 21 of [May 1999]. We now observe that $\partial L=\Lambda_{+} \sqcup \Lambda_{-}$and hence, from (3-1), we get that

$$
2 \chi(L)=\chi(\partial L)=\chi\left(\Lambda_{+}\right)+\chi\left(\Lambda_{-}\right) .
$$

Then we use Proposition 3.1 and rewrite (3-2) as

$$
2 \chi(L)=\chi\left(\Lambda_{+}\right)+\chi\left(\Lambda_{-}\right)=2(-1)^{-n / 2-1}\left(\operatorname{tb}\left(\Lambda_{+}\right)+\operatorname{tb}\left(\Lambda_{-}\right)\right) .
$$

From (3-3) it follows that

$$
\operatorname{tb}\left(\Lambda_{+}\right)+\operatorname{tb}\left(\Lambda_{-}\right)=(-1)^{n / 2+1} \chi(L) .
$$

This finishes the proof of Theorem 1.3 in the case when $n$ is even.

We now prove case (2) of the theorem. First we provide an alternate definition of the Thurston-Bennequin number, found in [Ekholm et al. 2005a].

Let $\Lambda$ be a closed, orientable, connected, chord-generic Legendrian submanifold of $\mathbb{R}^{2 n+1}$ and let $c$ be a Reeb chord of $\Lambda$ with end points $a$ and $b$ such that $z(a)>z(b)$. We define $V_{a}:=d \Pi\left(T_{a} \Lambda\right)$ and $V_{b}:=d \Pi\left(T_{b} \Lambda\right)$. Given an orientation on $\Lambda, V_{a}$ and $V_{b}$ are oriented $n$-dimensional transverse subspaces of $\mathbb{R}^{2 n}$. If the orientation of $V_{a} \oplus V_{b}$ agrees with that of $\mathbb{R}^{2 n}$, we say that the sign of $c$, denoted by $\operatorname{sign}(c)$, is +1 , otherwise we say that it is -1 . Then

$$
\operatorname{tb}(\Lambda)=\sum_{c} \operatorname{sign}(c),
$$

where the sum is taken over all Reeb chords $c$ of $\Lambda$.

The following proposition was proven using (3-5):

Proposition 3.2 [Ekholm et al. 2005b]. If $\Lambda \subset \mathbb{R}^{2 n+1}$ is a closed, orientable, connected, chord generic Legendrian submanifold,

$$
\operatorname{tb}(\Lambda)=(-1)^{((n-2)(n-1)) / 2} \sum_{c \in \mathscr{C}}(-1)^{|c|} .
$$

We now construct an exact Lagrangian filling of $\Lambda_{+}$. We do it the same way as in the proof of Theorem 1.2, namely $L_{\Lambda_{+}}$is obtained by gluing the positive end of $L_{\Lambda_{-}}$to the negative end of $L$ in the symplectization of $\mathbb{R}^{2 n+1}$. 
By using Proposition 3.2 and taking Euler characteristics of the long exact sequence (1-2), we get

$$
\operatorname{tb}\left(\Lambda_{+}\right)-\operatorname{tb}\left(\Lambda_{-}\right)=(-1)^{((n-2)(n-1)) / 2+1} \chi(L) .
$$

This finishes the proof of Theorem 1.3 when $n$ is odd.

Remark 3.3. When $n=1$ we can write (3-6) as

$$
\operatorname{tb}\left(\Lambda_{+}\right)-\operatorname{tb}\left(\Lambda_{-}\right)=-\chi(L),
$$

which coincides with the formula from Theorem 1.2 of [Chantraine 2010].

Remark 3.4. Observe that the condition of Theorem 1.3 in the case when $n$ is odd is much stronger than the condition of Theorem 1.3 in the case when $n$ is even. If $n$ is even, $\varnothing \prec_{L_{\Lambda_{-}}}^{\mathrm{ex}} \Lambda_{-}$and $\Lambda_{-} \prec_{L}^{\mathrm{ex}} \Lambda_{+}$, then, taking Euler characteristics of the long exact sequence (1-2) and using Proposition 3.2, we get that

$$
\operatorname{tb}\left(\Lambda_{+}\right)+\operatorname{tb}\left(\Lambda_{-}\right)=(-1)^{n / 2+1} \chi(L) .
$$

The proof of Theorem 1.3 can be easily modified to become a proof of the following remark.

Remark 3.5. Let $\Lambda$ be a closed, orientable Legendrian submanifold of $\mathbb{R}^{2 n+1}$.

(1) If $n$ is even and $\varnothing \prec_{L_{\Lambda}} \Lambda$,

$$
\operatorname{tb}(\Lambda)=(-1)^{n / 2+1} \chi\left(L_{\Lambda}\right) .
$$

(2) If $n$ is odd and $\varnothing \prec_{L_{\Lambda}}^{\mathrm{ex}} \Lambda$,

$$
\operatorname{tb}(\Lambda)=(-1)^{((n-2)(n-1)) / 2+1} \chi\left(L_{\Lambda}\right) .
$$

\section{Examples}

In this section, we describe a few examples of Lagrangian cobordisms. These examples are based on [Chantraine 2010; Ekholm et al. 2005b] and the work of Ekholm, Honda, and Kálmán [Ekholm et al. $\geq 2013$ ]. For the constructions of Lagrangian cobordisms based on the generating families technique, we refer to [Bourgeois et al. $\geq 2013$ ].

Example 4.1. Proof of Proposition 1.4. Let $\Lambda_{-}$and $\Lambda_{+} \subset \mathbb{R}^{2 n+1}$ be two closed, orientable Legendrian submanifolds which are Legendrian isotopic. Then there is a smooth isotopy of a closed manifold $\Lambda$ to $\mathbb{R}^{2 n+1}$ given by $\varphi: \Lambda \times[0,1] \rightarrow \mathbb{R}^{2 n+1}$ such that $\Lambda_{v}:=\varphi(\Lambda, v)$ is Legendrian for all $v \in[0,1], \Lambda_{-}=\Lambda_{0}$ and $\Lambda_{+}=$ $\Lambda_{1}$. We now construct $L$ such that $\Lambda_{-} \prec_{L}^{\text {ex }} \Lambda_{+}$. Observe that in the construction below one can omit the assumption that $\Lambda_{-}, \Lambda_{+}, L$ are connected. In the case of Legendrian knots in $\mathbb{R}^{3}$, the construction of $L$ was described in [Chantraine 2010, 
Theorem 1.1]. In our case, the construction of Chantraine can be described in the following way.

(1) Note that $\mathbb{R} \times \Lambda_{-}$is an exact Lagrangian submanifold of $\left(\mathbb{R} \times \mathbb{R}^{2 n+1}, d\left(e^{t} \alpha\right)\right)$.

(2) Theorem 2.6.2 of [Geiges 2008] implies that there is a compactly supported one-parameter family of contactomorphisms $f_{v}$ which realizes the isotopy $\left(\Lambda_{\nu}\right)_{v \in[0,1]}$.

(3) Proposition 2.2 from [Chantraine 2010] implies that a contactomorphism of $\mathbb{R}^{2 n+1}$ lifts to a Hamiltonian diffeomorphism of the symplectization

$$
\left(\mathbb{R} \times \mathbb{R}^{2 n+1}, d\left(e^{t} \alpha\right)\right) .
$$

(4) Let $H$ be a Hamiltonian on $\mathbb{R} \times \mathbb{R}^{2 n+1}$ whose flow realizes the lifts of $f_{v} \mathrm{~s}$. The existence of $H$ follows from (3). Following Chantraine, we construct

$$
H^{\prime}: \mathbb{R} \times \mathbb{R}^{2 n+1} \times[0,1] \rightarrow \mathbb{R}
$$

such that

$$
H^{\prime}(t, x, v)= \begin{cases}H(t, x, v) & \text { for } t>T \\ 0 & \text { for } t<-T\end{cases}
$$

Here $T \gg 0$.

(5) Let $\phi^{v}$ be the Hamiltonian flow of $H^{\prime}$. We now observe that $\phi^{1}\left(\mathbb{R} \times \Lambda_{-}\right)$ coincides with $\mathbb{R} \times \Lambda_{-}$near $-\infty$ and with $\mathbb{R} \times \Lambda_{+}$near $\infty$.

(6) Since $\mathbb{R} \times \Lambda_{-}$is exact and $\phi^{1}$ a Hamiltonian diffeomorphism, $L:=\phi^{1}\left(\mathbb{R} \times \Lambda_{-}\right)$ is exact.

Remark 4.2. Eliashberg and Gromov [1998] provided another proof of the fact that Legendrian isotopy implies Lagrangian cobordism.

Example 4.3. Proof of Proposition 1.5. The following construction is based on the front spinning method invented in [Ekholm et al. 2005b].

First we recall the notion of the front projection. The front projection is a map $\Pi_{F}$ from $\mathbb{R}^{2 n+1}$ to $\mathbb{R}^{n+1}$ defined by

$$
\Pi_{F}\left(x_{1}, y_{1}, \ldots, x_{n}, y_{n}, z\right)=\left(x_{1}, x_{2}, \ldots, x_{n}, z\right) .
$$

Let $\Lambda$ be a closed, orientable Legendrian submanifold of $\mathbb{R}^{2 n+1}$ parametrized by $f_{\Lambda}: \Lambda \rightarrow \mathbb{R}^{2 n+1}$. We write

$$
f_{\Lambda}(p)=\left(x_{1}(p), y_{1}(p), \ldots, x_{n}(p), y_{n}(p), z(p)\right)
$$

for $p \in \Lambda$. The front projection of $\Lambda$ is parametrized by $\Pi_{F} \circ f_{\Lambda}$, and we have

$$
\Pi_{F} \circ f_{\Lambda}(p)=\left(x_{1}(p), x_{2}(p), \ldots, x_{n}(p), z(p)\right) .
$$


Without loss of generality we can assume that $x_{1}(p)>0$ for all $p \in \Lambda$. We now embed $\mathbb{R}^{n+1}$ to $\mathbb{R}^{n+2}$ via

$$
\left(x_{1}, \ldots, x_{n}, z\right) \rightarrow\left(x_{0}=0, x_{1}, \ldots, x_{n}, z\right)
$$

and construct the suspension of $\Lambda$, denoted by $\Sigma \Lambda$, such that $\Pi_{F}(\Sigma \Lambda)$ is obtained from $\Pi_{F}(\Lambda)$ by rotating it around the subspace $x_{0}=x_{1}=0 . \Pi_{F}(\Sigma \Lambda)$ can be parametrized by $\left(x_{1}(p) \sin \theta, x_{1}(p) \cos \theta, x_{2}(p), \ldots, x_{n}(p), z(p)\right)$ with $\theta \in S^{1}$ and is the front projection of a Legendrian embedding $\Lambda \times S^{1} \rightarrow \mathbb{R}^{2 n+3}$. For the properties of $\Sigma \Lambda$ we refer to Lemma 4.16 of [Ekholm et al. 2005b].

Let $\Lambda_{-}$and $\Lambda_{+}$be two closed, orientable Legendrian submanifolds of $\mathbb{R}^{2 n+1}$ such that

$$
\Lambda_{ \pm} \subset\left\{\left(x_{1}, y_{1}, \ldots, x_{n}, y_{n}, z\right) \in \mathbb{R}^{2 n+1} \mid x_{1}>0\right\}
$$

and $\Lambda_{-} \prec_{L}^{\text {lag }} \Lambda_{+}$. Let $L$ be parametrized by $f_{L}: L \rightarrow \mathbb{R}^{2 n+2}$

$$
f_{L}(p)=\left(t(p), x_{1}(p), y_{1}(p), \ldots, x_{n}(p), y_{n}(p), z(p)\right) .
$$

Without loss of generality we assume that $x_{1}(p)>0$ for all $p$. (Formula (4-1) implies that

$$
\left\{f_{L}(p) \mid x_{1}(p) \leq 0\right\}
$$

is compact and we can translate $L$ so that $x_{1}(p)>0$ for all $p$.) Then we construct a Lagrangian cobordism from $\Sigma \Lambda_{-}$to $\Sigma \Lambda_{+}$that we call $\Sigma L$. We define $\Sigma L$ to be parametrized by

$$
f_{\Sigma L}: L \times S^{1} \rightarrow \mathbb{R} \times \mathbb{R}^{2 n+3}
$$

with

$$
\begin{aligned}
& f_{\Sigma L}(p, \theta) \\
& \quad=\left(t(p), x_{1}(p) \sin \theta, y_{1}(p) \sin \theta, x_{1}(p) \cos \theta, y_{1}(p) \cos \theta, x_{2}(p), \ldots, z(p)\right) .
\end{aligned}
$$

Here $p \in L$ and $\theta \in S^{1}$.

We now show that $\Sigma L$ is really a Lagrangian cobordism from $\Sigma \Lambda_{-}$to $\Sigma \Lambda_{+}$. Let

$$
\begin{aligned}
& \Lambda_{+}^{T_{L}}:=\left\{\left(x_{0}, \ldots, y_{n}, z\right) \mid\left(T_{L}, x_{0}, \ldots, y_{n}, z\right) \in f_{\Sigma L}(\Sigma L) \cap\left(\left\{T_{L}\right\} \times \mathbb{R}^{2 n+3}\right)\right\}, \\
& \Lambda_{-}^{T_{L}}:=\left\{\left(x_{0}, \ldots, y_{n}, z\right) \mid\left(-T_{L}, x_{0}, \ldots, y_{n}, z\right) \in f_{\Sigma L}(\Sigma L) \cap\left(\left\{-T_{L}\right\} \times \mathbb{R}^{2 n+3}\right)\right\} .
\end{aligned}
$$

From the definition of $T_{L}$, it follows that

$$
\begin{aligned}
f_{\Sigma L}(\Sigma L) \cap\left(\left[T_{L}, \infty\right) \times \mathbb{R}^{2 n+3}\right) & =\left[T_{L}, \infty\right) \times \Lambda_{+}^{T_{L}}, \\
f_{\Sigma L}(\Sigma L) \cap\left(\left(-\infty,-T_{L}\right] \times \mathbb{R}^{2 n+3}\right) & =\left(-\infty,-T_{L}\right] \times \Lambda_{-}^{T_{L}} .
\end{aligned}
$$


In addition, we observe that $\Lambda_{ \pm}^{T_{L}} \subset \mathbb{R}^{2 n+3}$ can be parametrized by

$$
f_{\Lambda_{ \pm}^{T_{L}}}: \Lambda_{ \pm} \times S^{1} \rightarrow \mathbb{R}^{2 n+3}
$$

such that

$f_{\Lambda_{ \pm}^{T_{L}}}(p, \theta)=\left(x_{1}(p) \sin \theta, y_{1}(p) \sin \theta, x_{1}(p) \cos \theta, y_{1}(p) \cos \theta, x_{2}(p), \ldots, z(p)\right)$.

Here $p \in \Lambda_{ \pm} \subset \partial L$ and $\theta \in S^{1}$. We now prove that $\Lambda_{ \pm}^{T_{L}}$ coincides with $\Sigma \Lambda_{ \pm}$. It is clear that $\Pi_{F}\left(\Lambda_{ \pm}^{T_{L}}\right)=\Pi_{F}\left(\Sigma \Lambda_{ \pm}\right)$. It remains to prove that $\Lambda_{ \pm}^{T_{L}}$ is a Legendrian submanifold of $\mathbb{R}^{2 n+3}$.

It is easy to see that

$$
\begin{aligned}
f_{\Lambda_{ \pm}}^{*}\left(d z-\sum_{i=0}^{n} y_{i} d x_{i}\right) & =d z(p)-\sum_{i=2}^{n} y_{i}(p) d x_{i}(p) \\
-y_{1}(p)\left(\sin ^{2} \theta+\cos ^{2} \theta\right) d x_{1}(p)+( & y_{1}(p) x_{1}(p) \sin \theta \cos \theta \\
& \left.-y_{1}(p) x_{1}(p) \sin \theta \cos \theta\right) d \theta .
\end{aligned}
$$

Since $\Lambda_{ \pm}$is a Legendrian submanifold of $\mathbb{R}^{2 n+1}$ and so $f_{\Lambda_{ \pm}}^{*}\left(d z-\sum_{i=1}^{n} y_{i} d x_{i}\right)=0$, we have

$$
y_{1}(p) d x_{1}(p)=d z(p)-\sum_{i=2}^{n} y_{i}(p) d x_{i}(p) .
$$

Hence (4-2) and (4-3) imply that

$$
f_{\Lambda_{ \pm}^{T_{L}}}^{*}\left(d z-\sum_{i=0}^{n} y_{i} d x_{i}\right)=0 .
$$

Since

$$
f_{\Lambda_{ \pm}}(p):=\left(x_{1}(p), \ldots, y_{n}(p), z(p)\right)
$$

where $p \in \Lambda_{ \pm} \subset \partial L$ is a parametrization of an embedded submanifold of dimension $n$, and $x_{1}(p)>0$ for $p \in \Lambda_{ \pm} \subset \partial L$, one easily sees that

$$
f_{\Lambda_{ \pm}^{T_{L}}}(p)=\left(x_{1}(p) \sin \theta, y_{1}(p) \sin \theta, x_{1}(p) \cos \theta, y_{1}(p) \cos \theta, x_{2}(p), \ldots, z(p)\right),
$$

where $p \in \Lambda_{ \pm}, \theta \in S^{1}$, is a parametrization of an embedded submanifold of dimension $n+1$. Thus, using (4-4), we see that $\Lambda_{ \pm}^{T_{L}}$ is an embedded Legendrian submanifold of $\mathbb{R}^{2 n+3}$ whose front projection coincides with $\Pi_{F}\left(\Sigma \Lambda_{ \pm}\right)$. Thus we get that $\Lambda_{ \pm}^{T_{L}}=\Sigma \Lambda_{ \pm}$.

We now note that 


$$
\begin{aligned}
& f_{\Sigma L}^{*}\left(d\left(e^{t}\left(d z-\sum_{i=0}^{n} y_{i} d x_{i}\right)\right)\right)=e^{t}\left(d t(p) \wedge d z(p)-\sum_{i=2}^{n} d y_{i}(p) \wedge d x_{i}(p)\right. \\
& -\sum_{i=2}^{n} y_{i}(p) d t(p) \wedge d x_{i}(p)-\left(y_{1}(p)\left(\sin ^{2} \theta+\cos ^{2} \theta\right) d t(p) \wedge d x_{1}(p)\right. \\
& +\left(\sin ^{2} \theta+\cos ^{2} \theta\right) d y_{1}(p) \wedge d x_{1}(p)+\left(\sin ^{2} \theta+\cos ^{2} \theta\right) x_{1}(p) y_{1}(p) d \theta \wedge d \theta \\
& +\left(y_{1}(p) x_{1}(p) \sin \theta \cos \theta-y_{1}(p) x_{1}(p) \sin \theta \cos \theta\right) d t(p) \wedge d \theta \\
& +\left(y_{1}(p) \sin \theta \cos \theta-y_{1}(p) \sin \theta \cos \theta\right) d \theta \wedge d x_{1}(p) \\
& \left.\left.+\left(x_{1}(p) \sin \theta \cos \theta-x_{1}(p) \sin \theta \cos \theta\right) d y_{1}(p) \wedge d \theta\right)\right)
\end{aligned}
$$

In addition, observe that

$$
\text { (4-6) } \begin{aligned}
e^{t}\left(d t(p) \wedge d z(p)-\sum_{i=2}^{n} d y_{i}(p) \wedge d x_{i}(p)-\sum_{i=2}^{n} y_{i}(p) d t(p) \wedge d x_{i}(p)\right) \\
=e^{t}\left(y_{1}(p) d t(p) \wedge d x_{1}(p)+d y_{1}(p) \wedge d x_{1}(p)\right) .
\end{aligned}
$$

Hence (4-5) and (4-6) imply that

$$
f_{\Sigma L}^{*}\left(d\left(e^{t}\left(d z-\sum_{i=0}^{n} y_{i} d x_{i}\right)\right)\right)=0
$$

Since

$$
f_{L}(p)=\left(t(p), x_{1}(p), y_{1}(p), \ldots, x_{n}(p), y_{n}(p), z(p)\right),
$$

where $p \in L$, is a parametrization of an embedded cobordism of dimension $n+1$ and $x_{1}(p)>0$ for $p \in L$, one easily sees that

$f_{\Sigma L}(p, \theta)$

$$
=\left(t(p), x_{1}(p) \sin \theta, y_{1}(p) \sin \theta, x_{1}(p) \cos \theta, y_{1}(p) \cos \theta, x_{2}(p), \ldots, z(p)\right),
$$

where $p \in L$ and $\theta \in S^{1}$, is a parametrization of an embedded cobordism of dimension $n+2$. Hence we use (4-7) and see that $\Sigma L$ is really an embedded Lagrangian cobordism from $\Sigma \Lambda_{-}$to $\Sigma \Lambda_{+}$.

We now assume that $\Lambda_{-} \prec_{L}^{\mathrm{ex}} \Lambda_{+}$. Then there is a function $h_{L} \in C^{\infty}\left(f_{L}(L), \mathbb{R}\right)$ such that

$$
d h_{L}=e^{t}\left(d z-\sum_{i=1}^{n} y_{i} d x_{i}\right)
$$

From a calculation similar to (4-2) it follows that

$$
f_{\Sigma L}^{*}\left(e^{t}\left(d z-\sum_{i=0}^{n} y_{i} d x_{i}\right)\right)=e^{t(p)}\left(d z(p)-\sum_{i=1}^{n} y_{i}(p) d x_{i}(p)\right) .
$$


Since $f_{\Sigma L}$ is an embedding, we can define $h_{\Sigma L} \in C^{\infty}\left(f_{\Sigma L}(\Sigma L), \mathbb{R}\right)$ by setting

$$
\left(f_{\Sigma L}^{*} h_{\Sigma L}\right)(p, \theta):=\left(f_{L}^{*} h_{L}\right)(p) .
$$

Hence we use (4-8) and get

$$
d\left(f_{\Sigma L}^{*} h_{\Sigma L}\right)=e^{t(p)}\left(d z(p)-\sum_{i=1}^{n} y_{i}(p) d x_{i}(p)\right)=f_{\Sigma L}^{*}\left(e^{t}\left(d z-\sum_{i=0}^{n} y_{i} d x_{i}\right)\right) .
$$

Therefore, since $f_{\Sigma L}$ is an embedding, (4-9) implies that

$$
d\left(h_{\Sigma L}\right)=e^{t}\left(d z-\sum_{i=0}^{n} y_{i} d x_{i}\right) .
$$

Hence, $\Sigma L$ is an exact Lagrangian cobordism.

Note that the proof of Proposition 1.5 can be easily modified to become a proof of the following remark.

Remark 4.4. Let $\Lambda$ be a closed, orientable Legendrian submanifolds of $\mathbb{R}^{2 n+1}$. If $\varnothing \prec_{L_{\Lambda}}^{\text {lag }} \Lambda$, there exists a Lagrangian filling $L_{\Sigma \Lambda}$ such that $\varnothing \prec_{L_{\Sigma \Lambda}}^{\text {lag }} \Sigma \Lambda$. In addition, if $\varnothing \prec_{L_{\Lambda}}^{\mathrm{ex}} \Lambda$, there exists an exact Lagrangian filling $L_{\Sigma \Lambda}$ such that $\varnothing \prec_{L_{\Sigma \Lambda}}^{\mathrm{ex}} \Sigma \Lambda$.

Before we discuss the next example, we briefly recall a few facts about exact Lagrangian cobordisms between Legendrian knots in $\mathbb{R}^{3}$.

Theorem 4.5 [Ekholm et al. $\geq 2013$; Ekholm et al. 2007]. There exists an exact Lagrangian cobordism for the following:

(1) Legendrian isotopy,

(2) 0-resolution at a contractible crossing in the Lagrangian projection,

(3) capping off $a \mathrm{tb}=-1$ unknot with a disk.

See Figure 1 for the 0-resolution on the Lagrangian projection.

Following Ekholm, Honda, and Kálmán, we say that a contractible crossing of $\Lambda$ is a crossing so that $z_{1}-z_{0}$ can be shrunk to zero without affecting the other crossings. (Here $z_{1}$ is the $z$-coordinate on the upper strand and $z_{0}$ is the $z$-coordinate on the lower strand.)

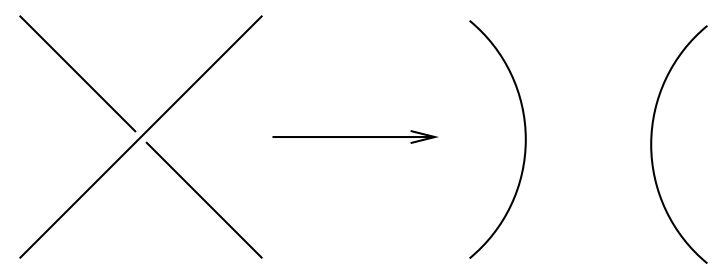

Figure 1. The 0-resolution on the Lagrangian projection. 
Remark 4.6. Chantraine [2010] proved the first part of Theorem 4.5.

Remark 4.7. Note that the second part of Theorem 4.5 can be proven using the model from Section 3.3 of [Rizell 2012].

Conjecture 4.8 [Ekholm et al. $\geq 2013$; Ekholm et al. 2007]. If $\varnothing \prec_{L_{\Lambda}}^{\mathrm{ex}} \Lambda$, then $L_{\Lambda}$ is obtained by stacking exact Lagrangians cobordisms described in Theorem 4.5.

Example 4.9. Proof of Proposition 1.6. We now use Example 4.3 to get infinitely many pairs of exact Lagrangian cobordant and not pairwise Legendrian isotopic Legendrian $n$-tori in $\mathbb{R}^{2 n+1}$. We first recall that Theorem 4.5 says that 0 -resolution at a contractible crossing in the Lagrangian projection can be realized as an exact Lagrangian cobordism. Let $T_{2 k+1}$ be the Legendrian torus knot from Example 4.18 of [Ekholm et al. 2005b]; see Figure 2 for the Lagrangian projection of $T_{2 k+1}$. One observes that all the crossings in the middle part of the Lagrangian projection are contractible (see [Ekholm et al. 2007] for the case of $T_{3}$ ) and hence one can get $T_{2 k-1}$ from $T_{2 k+1}$ by contracting $c_{2 k+1}$ and then $c_{2 k}$. Let $L_{2 k}^{2 k+1}$ be an exact Lagrangian cobordism which corresponds to the 0 -resolution at $c_{2 k+1}$ and $L_{2 k-1}^{2 k}$ an exact Lagrangian cobordism from $T_{2 k-1}$ to $T_{2 k}$ which corresponds to the resolution of $c_{2 k}$. Then we stack $L_{2 k}^{2 k+1}$ and $L_{2 k-1}^{2 k}$ and get an exact Lagrangian cobordism that we call $L_{2 k-1}^{2 k+1}$ such that

$$
T_{2 k-1} \prec \underset{L_{2 k-1}^{2 k+1}}{\mathrm{ex}} T_{2 k+1} .
$$

If we stack $L_{2 i-1}^{2 i+1} \mathrm{~s}$ we get an exact Lagrangian cobordism $L_{2 j+1}^{2 k+1}$ such that

$$
T_{2 j+1} \underset{L_{2 j+1}^{2 k+1}}{\mathrm{ex}_{2 k+1}} T_{2 k+1}
$$

for $k>j$. We use the construction described in Example 4.3 and get

$$
\Sigma^{n} T_{2 j+1} \prec \sum_{\Sigma^{n} L_{2 j+1}^{2 k+1}}^{\mathrm{ex}} \Sigma^{n} T_{2 k+1}
$$

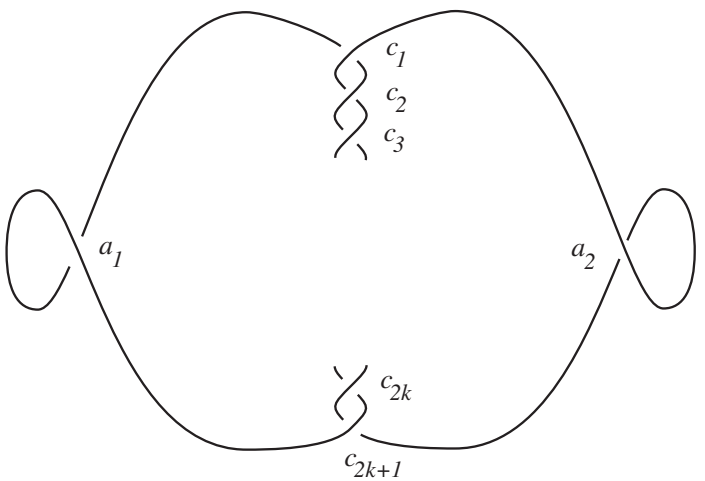

Figure 2. The knot $T_{2 k+1}$; cf. Figure 13 of [Ekholm et al. 2005b] . 
for $k>j$. We now recall that Ekholm, Etnyre, and Sullivan [Ekholm et al. 2005b, Theorem 4.19] proved that $\Sigma^{n} T_{2 j+1}$ is not Legendrian isotopic to $\Sigma^{n} T_{2 k+1}$ for $k>j+1$ and $j \in \mathbb{N}$.

Hence we get infinitely many pairs of exact Lagrangian cobordant and not pairwise Legendrian isotopic Legendrian $n$-tori in $\mathbb{R}^{2 n+1}$.

Remark 4.10. Given $n \geq 1$, we observe that Theorem 4.19 of [Ekholm et al. 2005b] implies that all the Legendrian $n$-tori from Proposition 1.6 are not distinguished by the classical invariants.

\section{Acknowledgements}

The author is deeply grateful to Baptiste Chantraine, Vincent Colin, Olivier Collin, Octav Cornea, Tobias Ekholm, Yakov Eliashberg, John Etnyre, Paolo Ghiggini, Ko Honda, Clément Hyvrier, Georgios Dimitroglou Rizell, Joshua Sabloff, Lisa Traynor and Vera Vértesi for helpful conversations and interest in his work. Specifically, we thank Joshua Sabloff and Lisa Traynor for pointing out the way to get the second long exact sequence in Theorem 1.2.

In addition, the author is grateful to the referee of an earlier version of this paper for many valuable comments and suggestions.

\section{References}

[Abouzaid and Seidel 2010] M. Abouzaid and P. Seidel, "An open string analogue of Viterbo functoriality", Geom. Topol. 14:2 (2010), 627-718. MR 2011g:53190 Zbl 1195.53106

[Bennequin 1983] D. Bennequin, "Entrelacements et équations de Pfaff", pp. 87-161 in Third Schnepfenried geometry conference, Vol. 1 (Schnepfenried, 1982), Astérisque 107, Soc. Math. France, Paris, 1983. MR 86e:58070 Zbl 0573.58022

[Bourgeois et al. $\geq 2013$ ] F. Bourgeois, J. Sabloff, and L. Traynor, "Lagrangian cobordisms via generating families with applications to Legendrian geography and botany", in preparation.

[Chantraine 2010] B. Chantraine, "Lagrangian concordance of Legendrian knots", Algebr. Geom. Topol. 10:1 (2010), 63-85. MR 2011f:57049 Zbl 1203.57010

[Chekanov 2002] Y. Chekanov, "Differential algebra of Legendrian links", Invent. Math. 150:3 (2002), 441-483. MR 2003m:53153 Zbl 1029.57011

[Ekholm 2008] T. Ekholm, "Rational symplectic field theory over $\mathbb{Z}_{2}$ for exact Lagrangian cobordisms”, J. Eur. Math. Soc. (JEMS) 10:3 (2008), 641-704. MR 2009g:53130 Zbl 1154.57020

[Ekholm 2012] T. Ekholm, "Rational SFT, linearized Legendrian contact homology, and Lagrangian Floer cohomology", pp. 109-145 in Perspectives in analysis, geometry, and topology, edited by I. Itenberg et al., Progr. Math. 296, Birkhäuser/Springer, New York, 2012. MR 2884034 Zbl 0608 2164

[Ekholm et al. 2005a] T. Ekholm, J. Etnyre, and M. Sullivan, "The contact homology of Legendrian submanifolds in $\mathbb{R}^{2 n+1}$, J. Differential Geom. 71:2 (2005), 177-305. MR 2007f:53115 Zbl 1103.53048

[Ekholm et al. 2005b] T. Ekholm, J. Etnyre, and M. Sullivan, "Non-isotopic Legendrian submanifolds in $\mathbb{R}^{2 n+1}$, J. Differential Geom. 71:1 (2005), 85-128. MR 2006i:53119 Zbl 1098.57013 
[Ekholm et al. 2007] T. Ekholm, K. Honda, and T. Kálmán, "Invariants of exact Lagrangian cobordisms", lecture slides, 2007, http://www.crm.umontreal.ca/Stanford2007/pdf/HondaSlides.pdf.

[Ekholm et al. $\geq 2013]$ T. Ekholm, K. Honda, and T. Kálmán, "Invariants of exact Lagrangian cobordisms", in preparation.

[Eliashberg 1990] Y. Eliashberg, "Topological characterization of Stein manifolds of dimension > 2”, Internat. J. Math. 1:1 (1990), 29-46. MR 91k:32012 Zbl 0699.58002

[Eliashberg and Gromov 1998] Y. Eliashberg and M. Gromov, "Lagrangian intersection theory: finite-dimensional approach", pp. 27-118 in Geometry of differential equations, edited by A. Khovanskiŭ et al., Amer. Math. Soc. Transl. Ser. 2 186, Amer. Math. Soc., Providence, RI, 1998. MR 2002a:53102 Zbl 0919.58015

[Eliashberg et al. 2000] Y. Eliashberg, A. Givental, and H. Hofer, "Introduction to symplectic field theory", Geom. Funct. Anal. Special Volume, Part II (2000), 560-673. GAFA 2000 (Tel Aviv, 1999). MR 2002e:53136 Zbl 0989.81114

[Fukaya et al. 2009] K. Fukaya, P. Seidel, and I. Smith, "The symplectic geometry of cotangent bundles from a categorical viewpoint", pp. 1-26 in Homological mirror symmetry, edited by A. Kapustin et al., Lecture Notes in Phys. 757, Springer, Berlin, 2009. MR 2011c:53213 Zbl 1163.53344

[Geiges 2008] H. Geiges, An introduction to contact topology, Cambridge Studies in Advanced Mathematics 109, Cambridge University Press, 2008. MR 2008m:57064 Zbl 1153.53002

[May 1999] J. P. May, A concise course in algebraic topology, University of Chicago Press, 1999. MR 2000h:55002 Zbl 0923.55001

[Rizell 2012] G. D. Rizell, "Legendrian ambient surgery and Legendrian contact homology", preprint, 2012. arXiv 1205.5544

[Sabloff and Traynor 2010] J. M. Sabloff and L. Traynor, "Obstructions to the existence and squeezing of Lagrangian cobordisms”, J. Topol. Anal. 2:2 (2010), 203-232. MR 2011g:53185 Zbl 1210. 57026

[Sabloff and Traynor 2011] J. Sabloff and L. Traynor, "Obstructions to Lagrangian cobordisms between Legendrian submanifolds", preprint, 2011. arXiv 1109.5660

[Tabachnikov 1988] S. L. Tabachnikov, "An invariant of a submanifold that is transversal to a distribution", Uspekhi Mat. Nauk 43:3(261) (1988), 193-194. In Russian; translated in Russian Math. Surveys 43:3 (1998), 225-226. MR 89m:58077

Received December 6, 2011. Revised July 16, 2012.

\section{ROMAN GOLOVKO}

DÉPARTEMENT DE MATHÉMATIQUES

UNIVERSITÉ LIBRE DE BRUXELLES

CP 218, BOULEVARD DU TRIOMPHE

1050 BRUXELLES

BELGIUM

rgolovko@ulb.ac.be 


\title{
PACIFIC JOURNAL OF MATHEMATICS
}

\author{
msp.org/pjm
}

Founded in 1951 by E. F. Beckenbach (1906-1982) and F. Wolf (1904-1989)

\section{EDITORS}

V. S. Varadarajan (Managing Editor)

Department of Mathematics

University of California

Los Angeles, CA 90095-1555

pacific@math.ucla.edu

Paul Balmer

Department of Mathematics

University of California

Los Angeles, CA 90095-1555

balmer@math.ucla.edu

Daryl Cooper

Department of Mathematics

University of California

Santa Barbara, CA 93106-3080 cooper@math.ucsb.edu

Jiang-Hua $\mathrm{Lu}$

Department of Mathematics

The University of Hong Kong

Pokfulam Rd., Hong Kong jhlu@maths.hku.hk
Don Blasius

Department of Mathematics University of California

Los Angeles, CA 90095-1555

blasius@math.ucla.edu

Robert Finn

Department of Mathematics Stanford University

Stanford, CA 94305-2125

finn@math.stanford.edu

Sorin Popa

Department of Mathematics

University of California

Los Angeles, CA 90095-1555

popa@math.ucla.edu

Paul Yang

Department of Mathematics

Princeton University

Princeton NJ 08544-1000

yang@math.princeton.edu

\section{PRODUCTION}

Silvio Levy, Scientific Editor, production@msp.org

\section{SUPPORTING INSTITUTIONS}

ACADEMIA SINICA, TAIPEI

CALIFORNIA INST. OF TECHNOLOGY

INST. DE MATEMÁTICA PURA E APLICADA

KEIO UNIVERSITY

MATH. SCIENCES RESEARCH INSTITUTE

NEW MEXICO STATE UNIV.

OREGON STATE UNIV.

\author{
STANFORD UNIVERSITY \\ UNIV. OF BRITISH COLUMBIA \\ UNIV. OF CALIFORNIA, BERKELEY \\ UNIV. OF CALIFORNIA, DAVIS \\ UNIV. OF CALIFORNIA, LOS ANGELES \\ UNIV. OF CALIFORNIA, RIVERSIDE \\ UNIV. OF CALIFORNIA, SAN DIEGO \\ UNIV. OF CALIF., SANTA BARBARA
}

\author{
Vyjayanthi Chari \\ Department of Mathematics \\ University of California \\ Riverside, CA 92521-0135 \\ chari@math.ucr.edu \\ Kefeng Liu \\ Department of Mathematics \\ University of California \\ Los Angeles, CA 90095-1555 \\ liu@math.ucla.edu \\ Jie Qing \\ Department of Mathematics \\ University of California \\ Santa Cruz, CA 95064 \\ qing@cats.ucsc.edu
}

These supporting institutions contribute to the cost of publication of this Journal, but they are not owners or publishers and have no responsibility for its contents or policies.

See inside back cover or msp.org/pjm for submission instructions.

The subscription price for 2013 is US \$400/year for the electronic version, and \$485/year for print and electronic.

Subscriptions, requests for back issues and changes of subscribers address should be sent to Pacific Journal of Mathematics, P.O. Box 4163, Berkeley, CA 94704-0163, U.S.A. The Pacific Journal of Mathematics is indexed by Mathematical Reviews, Zentralblatt MATH, PASCAL CNRS Index, Referativnyi Zhurnal, Current Mathematical Publications and the Science Citation Index.

The Pacific Journal of Mathematics (ISSN 0030-8730) at the University of California, c/o Department of Mathematics, 798 Evans Hall \#3840, Berkeley, CA 94720-3840, is published monthly except July and August. Periodical rate postage paid at Berkeley, CA 94704, and additional mailing offices. POSTMASTER: send address changes to Pacific Journal of Mathematics, P.O. Box 4163, Berkeley, CA 94704-0163.

PJM peer review and production are managed by EditFLOW ${ }^{\circledR}$ from Mathematical Sciences Publishers.

PUBLISHED BY

mathematical sciences publishers

nonprofit scientific publishing

http://msp.org/

(C) 2013 Mathematical Sciences Publishers 


\section{PACIFIC JOURNAL OF MATHEMATICS}

Volume $261 \quad$ No. $1 \quad$ January 2013

Hierarchies and compatibility on Courant algebroids

Paulo Antunes, Camille Laurent-GengouX and

JoANA M. NunES DA COSTA

A new characterization of complete linear Weingarten hypersurfaces in real 33 space forms

Cícero P. Aquino, Henrique F. DE Lima and

MARCO A. L. VELÁSQUEZ

Calogero-Moser versus Kazhdan-Lusztig cells

CÉDRIC BONNAFÉ and RAPHAËL ROUQUIER

Coarse median spaces and groups

BRIAN H. BOWDITCH

Geometrization of continuous characters of $\mathbb{Z}_{p}^{\times}$

CLIFTON CUNNINGHAM and MASOUd KAMGARPOUR

A note on Lagrangian cobordisms between Legendrian submanifolds of $\mathbb{R}^{2 n+1}$

\section{ROMAN GOLOVKO}

On slope genera of knotted tori in 4-space

Yi LIU, Yi Ni, HoNGBIN SUN and SHICHENG WANG

Formal groups of elliptic curves with potential good supersingular reduction

$$
\text { ÁlVARO LOZANO-ROBLEDO }
$$

Codimension-one foliations calibrated by nondegenerate closed 2-forms

DAVID MARTínez TORRES

The trace of Frobenius of elliptic curves and the $p$-adic gamma function

DERMOT MCCARTHY

$(D N)-(\Omega)$-type conditions for Fréchet operator spaces 